\title{
ON TYPICAL MARKOV OPERATORS ACTING ON BOREL MEASURES
}

TOMASZ SZAREK

Received 20 February 2004

It is proved that, in the sense of Baire category, almost every Markov operator acting on Borel measures is asymptotically stable and the Hausdorff dimension of its invariant measure is equal to zero.

\section{Introduction}

Generic properties of different objects (functions, sets, measures, and many others) have been studied for a long time (see $[1,2,3,4,5,7,8,9,10,13,15,16])$. We say that some property is generic (or typical) if the subset of all elements satisfying this property is residual. Recall that a subset of a complete metric space is residual if its complement can be represented as a countable union of nowhere dense sets.

Generic properties of Markov operators have been recently examined by Lasota and Myjak [11, 12]. Indeed, they have shown that the typical Markov operator corresponding to an iterated function system is asymptotically stable and its invariant measure is singular with respect to the Lebesgue measure (see [12]). This result has been recently extended to learning systems and stochastic perturbed dynamical systems (see $[17,18])$. In [14], a more general result has been proved. Namely, most of the Markov operators in the class of all Markov operators acting on Borel measures in $\mathbb{R}^{d}$ are asymptotically stable and have a singular stationary measure.

Let $(X, \rho)$ be a complete and separable metric space. By $B(x, r)$ we denote the open ball with center $x$ and radius $r>0$. Given a set $A \subset X$ and number $r>0$, we denote by $\operatorname{diam} A$ the diameter of the set $A$ and by $B(A, r)$ the $r$-neighbourhood of the set $A$, that is,

$$
B(A, r)=\{x \in X: \rho(x, A)<r\}
$$

where $\rho(x, A)=\inf \{\rho(x, y): y \in A\}$.

By $\mathscr{B}(X)$ we denote the $\sigma$-algebra of all Borel subsets of $X$. By $M$ we denote the family of all finite Borel measures on $X$, by $M_{1}$ the space of all $\mu \in M$ such that $\mu(X)=1$, and by $M_{s}=\left\{\mu_{1}-\mu_{2}: \mu_{1}, \mu_{2} \in M\right\}$ the space of all finite signed Borel measures on $X$. 
Given $\mu \in M$, we define the support of $\mu$ by the formula

$$
\operatorname{supp} \mu=\{x \in X: \mu(B(x, r))>0 \text { for every } r>0\} .
$$

As usual, by $C(X)$ we denote the subspace of all bounded continuous functions. We consider this space with the supremum norm.

For $f \in C(X)$ and $\mu \in \mathcal{M}_{s}$, we will write

$$
\langle f, \mu\rangle=\int_{X} f(x) \mu(d x)
$$

We admit that $M_{s}$ is endowed with the Fortet-Mourier norm (see [6]) given by

$$
\|\mu\|_{\mathscr{F}}=\sup \{|\langle f, \mu\rangle|: f \in \mathscr{F}\} \quad \text { for } \mu \in M_{s},
$$

where $\mathscr{F}$ is the set of all $f \in C(X)$ such that $|f(x)| \leq 1$ and $|f(x)-f(y)| \leq \rho(x, y)$ for $x, y \in X$.

An operator $P: \mathcal{M} \rightarrow \mathcal{M}$ is called a Markov operator if it satisfies the following conditions:

(i) positive linearity:

$$
P\left(\lambda_{1} \mu_{1}+\lambda_{2} \mu_{2}\right)=\lambda_{1} P \mu_{1}+\lambda_{2} P \mu_{2}
$$

for $\lambda_{1}, \lambda_{2} \geq 0$ and $\mu_{1}, \mu_{2} \in \mathcal{M}$,

(ii) preservation of measures:

$$
P \mu(X)=\mu(X) \quad \text { for } \mu \in M
$$

A measure $\mu_{*}$ is called invariant (or stationary) with respect to $P$ if $P \mu_{*}=\mu_{*}$. A Markov operator $P$ is called asymptotically stable if there exists a stationary measure $\mu_{*} \in M_{1}$ such that

$$
\lim _{n \rightarrow \infty}\left\|P^{n} \mu-\mu_{*}\right\|_{\mathscr{F}}=0
$$

for every $\mu \in M_{1}$.

Let $\mathscr{P}$ denote the set of all continuous Markov operators $P: M \rightarrow M$, where $\mathcal{M}$ is endowed with the Fortet-Mourier metric. In this space, we introduce

$$
\hat{\rho}(P, Q)=\sup _{\mu \in M_{1}}\|P \mu-Q \mu\|_{\mathscr{F}}
$$

Clearly $\hat{\rho}$ is a distance and $\mathscr{P}$ with this distance is a complete metric space.

For $A \subset X$ and $s, \delta>0$, define

$$
\begin{gathered}
\mathscr{H}_{\delta}^{s}(A)=\inf \left\{\sum_{i=1}^{\infty}\left(\operatorname{diam} U_{i}\right)^{s}: A \subset \bigcup_{i=1}^{\infty} U_{i} \text { and } \operatorname{diam} U_{i} \leq \delta\right\}, \\
\mathscr{H}^{s}(A)=\lim _{\delta \rightarrow 0} \mathscr{H}_{\delta}^{s}(A) .
\end{gathered}
$$


The restriction of $\mathscr{H}^{s}$ to the $\sigma$-algebra of $\mathscr{H}^{s}$-measurable sets is called the Hausdorff sdimensional measure. Note that all Borel sets are $\mathscr{H}^{s}$-measurable. The value

$$
\operatorname{dim}_{H} A=\inf \left\{s>0: \mathscr{H}^{s}(A)=0\right\}
$$

is called the Hausdorff dimension of the set $A$. As usual, we admit inf $\varnothing=+\infty$.

The Hausdorff dimension of a measure $\mu \in M_{1}$ is defined by the formula

$$
\operatorname{dim}_{H} \mu=\inf \left\{\operatorname{dim}_{H} A: A \in \mathscr{B}(X), \mu(A)=1\right\} .
$$

We are in a position to formulate the main result of our note.

Theorem 1.1. Let $\mathscr{P}_{0}$ denote the set of all $P \in \mathscr{P}$ such that $P$ is asymptotically stable and its invariant measure $\mu_{P} \in M_{1}$ satisfies $\operatorname{dim}_{H}\left(\mu_{P}\right)=0$ and $\operatorname{supp} \mu_{P}=X$. Then $\mathscr{P}_{0}$ is residual in $\mathscr{P}$.

\section{Auxiliary results}

In this section, we recall auxiliary results which are useful in the proof of the main theorem. Lemma 2.1 has been already proved in [19]. On the other hand, Lemma 2.2 has been used in [14]. Since the proofs of both lemmas may be easily presented here, they are included in this section.

LeMma 2.1. Let $\mu_{1}, \mu_{2} \in M_{1}$ and $\varepsilon>0$. If $\left\|\mu_{1}-\mu_{2}\right\|_{\mathscr{F}} \leq \varepsilon^{2}$, then

$$
\mu_{1}(B(A, \varepsilon)) \geq \mu_{2}(A)-\varepsilon
$$

for every Borel set $A \subset X$.

Proof. Consider the function $f: X \rightarrow[0, \varepsilon]$ given by the formula

$$
f(x)=\max \{\varepsilon-\rho(A, x), 0\} .
$$

Since $f \in \mathscr{F}$ and $f(x)=0$ for $x \notin B(A, \varepsilon), f(x)=\varepsilon$ for $x \in A$, we have

$$
\varepsilon \mu_{2}(A)-\varepsilon \mu_{1}(B(A, \varepsilon)) \leq \int_{X} f d \mu_{2}-\int_{X} f d \mu_{1} \leq\left\|\mu_{2}-\mu_{1}\right\|_{\mathscr{F}} \leq \varepsilon^{2},
$$

whence the statement of Lemma 2.1 follows.

Lemma 2.2. Let $P \in \mathscr{P}$. Assume that there exist $\alpha \in(0,1)$ and $n_{0} \in \mathbb{N}$ such that for every $\mu_{1}, \mu_{2} \in M_{1}$, it can be found $\mu_{0} \in M_{1}$ satisfying

$$
P^{n_{0}} \mu_{i} \geq \alpha \mu_{0} \quad \text { for every } i=1,2 .
$$

Then $P$ is asymptotically stable.

Proof. Fix $\mu_{1}, \mu_{2} \in M_{1}$. Let $\alpha \in(0,1)$ and $n_{0}$ be such as in the statement of lemma. Let $\mu_{0} \in M_{1}$ be such that (2.4) holds. Observe that

$$
P^{n_{0}} \mu_{i}=\alpha \mu_{0}+(1-\alpha) \tilde{\mu}_{i}
$$


where

$$
\tilde{\mu}_{i}=\frac{1}{(1-\alpha)}\left(P^{n_{0}} \mu_{i}-\alpha \mu_{0}\right) \quad \text { for } i=1,2
$$

Hence,

$$
\left\|P^{n_{0}} \mu_{2}-P^{n_{0}} \mu_{1}\right\|_{\mathscr{F}}=(1-\alpha)\left\|\tilde{\mu}_{1}-\tilde{\mu}_{2}\right\|_{\mathscr{F}} \leq 2(1-\alpha) .
$$

By induction argument, we easily check that

$$
\left\|P^{k n_{0}} \mu_{1}-P^{k n_{0}} \mu_{2}\right\|_{\mathscr{F}} \leq 2(1-\alpha)^{k}
$$

for every $\mu_{1}, \mu_{2} \in M_{1}$ and $k \in \mathbb{N}$. Thus $\left(P^{n} \mu\right)_{n \geq 1}, \mu \in M_{1}$, satisfies the Cauchy condition. Since $\left(M_{1},\|\cdot\|_{\mathscr{F}}\right)$ is complete and $P$ is continuous, it follows immediately that $P$ admits an invariant measure. Finally, by $(2.8) P$ is asymptotically stable.

Lemma 2.3. Let $\mathscr{P}_{x}, x \in X$, denote the set of all $Q \in \mathscr{P}$ such that

(i) $Q$ is asymptotically stable and $x \in \operatorname{supp} \mu_{Q}$, where $\mu_{Q}$ is invariant for $Q$;

(ii) for every $n \in \mathbb{N}$, there exists $r \in(0,1 / n)$ such that

$$
\frac{\log \mu_{Q}(B(y, r))}{\log r} \leq \frac{1}{n} \quad \forall y \in \operatorname{supp} \mu_{Q} ;
$$

(iii) there exists $\alpha_{Q} \in(0,1)$ such that

$$
\left\|Q^{n} \mu_{1}-Q^{n} \mu_{2}\right\|_{\mathscr{F}} \leq 2\left(1-\alpha_{Q}\right)^{n}
$$

for every $\mu_{1}, \mu_{2} \in M_{1}$ and $n \in \mathbb{N}$. Then $\mathscr{P}_{x}$ is dense in $\mathscr{P}$.

Proof. Fix $x \in X, P \in \mathscr{P}$, and $\varepsilon>0$. Let $\left\{x_{m}\right\}_{m \geq 1}$ be a dense subset of $X$. Then

$$
X=\bigcup_{i=1}^{\infty} B\left(x_{i}, \frac{\varepsilon}{2}\right)
$$

Define the sets

$$
\begin{gathered}
D_{1}=B\left(x_{1}, \frac{\varepsilon}{2}\right), \\
D_{i}=B\left(x_{i}, \frac{\varepsilon}{2}\right) \backslash \bigcup_{j=1}^{i-1} B\left(x_{j}, \frac{\varepsilon}{2}\right), \quad i=2,3, \ldots
\end{gathered}
$$

The sets $D_{i}, i \geq 1$, are disjoint and cover $X$. Obviously diam $D_{i} \leq \varepsilon, i=1,2, \ldots$ Consider the operator $\tilde{Q}: \mathcal{M} \rightarrow \mathcal{M}$ given by

$$
\widetilde{Q} \mu=\sum_{i=1}^{\infty} P \mu\left(D_{i}\right) \delta_{x_{i}} \quad \text { for } \mu \in M_{1},
$$

where $\delta_{x}$ means the $\delta$-Dirac measure at $x$. Clearly $\widetilde{Q}$ is a Markov operator. 
Now fix an $x \in X$ and consider the operator $Q: \mathcal{M} \rightarrow \mathcal{M}$ given by

$$
Q=\left(1-\frac{\varepsilon}{4}\right) \widetilde{Q}+\frac{\varepsilon}{4} \Delta_{x}
$$

where $\Delta_{x}: \mathcal{M} \rightarrow \mathcal{M}, \Delta_{x}(\mu)=\mu(X) \delta_{x}$. Observe that $Q$ is a Markov operator as well. Moreover, from Lemma 2.1, it follows that $Q$ is asymptotically stable. Further, observe that $x \in \operatorname{supp} \mu_{Q}$. Since $\mu_{Q}$ is a purely atomic measure, condition (ii) holds. Finally, from the proof of Lemma 2.2, it follows that condition (iii) is satisfied and $\hat{\rho}(P, Q)<3 \varepsilon / 2$.

Using a standard Vitali argument, one can prove the following lemma.

Lemma 2.4. Let $\mu \in M_{1}$ and $Y \subset X$ be compact. If

$$
\liminf _{r \rightarrow 0} \frac{\log \mu(B(x, r))}{\log r}=0 \quad \text { for } x \in Y,
$$

then $\operatorname{dim}_{H} Y=0$.

The proof of the above lemma can be found in the literature under slightly weaker assumptions. Namely, it has been shown that if $\lim _{r \rightarrow 0}(\log \mu(B(x, r))) / \log r=0$ for all $x$ of some compact set $Y$, then $\operatorname{dim}_{H} Y=0$ (see [20]). For the convenience of the readers, we will give the proof of the lemma.

Proof. Fix $s>0$. Choose $\tilde{s} \in(0, s)$. We will show that $\mathscr{H}^{\tilde{s}}(Y)<\infty$. To do this, fix $\delta>0$. Since $Y$ is compact, we can choose a finite sequence of points $\left(x_{1}, \ldots, x_{m}\right), m \in \mathbb{N}$, such that

$$
\begin{gathered}
Y \subset \bigcup_{i=1}^{m} B\left(x_{i}, r_{i}\right), \\
\frac{\log \mu\left(B\left(x_{i}, r_{i}\right)\right)}{\log r_{i}} \leq \tilde{s},
\end{gathered}
$$

where $r_{i}<\min \{1, \delta / 6\}$ for $i=\{1, \ldots, m\}$. Without loss of generality, we may assume that

$$
r_{1} \geq r_{2} \geq \cdots \geq r_{m}
$$

By induction we define sequences $\left(y_{1}, \ldots, y_{\tilde{m}}\right)$ and $\left(\tilde{r}_{1}, \ldots, \tilde{r}_{\tilde{m}}\right)$ for some $\tilde{m} \in \mathbb{N}$ in the following way:

$$
y_{1}=x_{1}, \quad \tilde{r}_{1}=r_{1} .
$$

If we have chosen the sequences $\left(y_{1}, \ldots, y_{n}\right),\left(\tilde{r}_{1}, \ldots, \tilde{r}_{n}\right)$ and

$$
\left\{j \in\{1, \ldots, m\}: B\left(x_{j}, r_{j}\right) \cap B\left(y_{k}, \tilde{r}_{k}\right)=\varnothing \text { for } k \in\{1, \ldots, n\}\right\} \neq \varnothing \text {, }
$$

then

$$
y_{n+1}=x_{i}, \quad \tilde{r}_{n+1}=r_{i},
$$


where

$$
i=\min \left\{j \in\{1, \ldots, m\}: B\left(x_{j}, r_{j}\right) \cap B\left(y_{k}, \tilde{r}_{k}\right)=\varnothing \text { for } k \in\{1, \ldots, n\}\right\} .
$$

If

$$
\left\{j \in\{1, \ldots, m\}: B\left(x_{j}, r_{j}\right) \cap B\left(y_{k}, \tilde{r}_{k}\right)=\varnothing \text { for } k \in\{1, \ldots, n\}\right\}=\varnothing
$$

then we set $\tilde{m}=n$.

By the above we obtain

$$
B\left(y_{i}, \tilde{r}_{i}\right) \cap B\left(y_{j}, \tilde{r}_{j}\right)=\varnothing \quad \text { for } i \neq j, i, j \in\{1, \ldots, \tilde{m}\} .
$$

Thus

$$
\sum_{i=1}^{\tilde{m}} \mu\left(B\left(y_{i}, \tilde{r}_{i}\right)\right) \leq \mu(X)=1 .
$$

Moreover, it may be shown that

$$
Y \subset \bigcup_{i=1}^{\tilde{m}} B\left(y_{i}, 3 \tilde{r}_{i}\right) .
$$

For this purpose, fix $y \in Y$. Let $j \in\{1, \ldots, m\}$ be such that $y \in B\left(x_{j}, r_{j}\right)$. If $x_{j}=y_{j_{0}}$ for some $j_{0} \in\{1, \ldots, \tilde{m}\}$, then

$$
y \in \bigcup_{i=1}^{\tilde{m}} B\left(y_{i}, \tilde{r}_{i}\right) \subset \bigcup_{i=1}^{\tilde{m}} B\left(y_{i}, 3 \tilde{r}_{i}\right) .
$$

On the other hand, if $x_{j} \notin\{1, \ldots, \tilde{m}\}$, then there exists $k \in\{1, \ldots, \tilde{m}\}$ such that

$$
B\left(y_{k}, \tilde{r}_{k}\right) \cap B\left(x_{j}, r_{j}\right) \neq \varnothing
$$

and $\tilde{r}_{k} \geq r_{j}$. Therefore,

$$
B\left(x_{j}, r_{j}\right) \subset B\left(y_{k}, 3 \tilde{r}_{k}\right)
$$

and consequently

$$
y \in \bigcup_{i=1}^{\tilde{m}} B\left(y_{i}, 3 \tilde{r}_{i}\right)
$$

From (2.17) and (2.25), it follows that

$$
\sum_{i=1}^{\tilde{m}} \tilde{r}_{i}^{\tilde{s}} \leq 1
$$


Since $6 \tilde{r}_{i} \leq 6 r_{1}<\delta$ and $(2.17)$ holds, we have

$$
\mathcal{H}_{\delta}^{\tilde{s}}(Y) \leq \sum_{i=1}^{\tilde{m}} 6^{\tilde{s}} \tilde{r}_{i}^{\tilde{s}} \leq 6^{\tilde{s}} .
$$

Letting $\delta \rightarrow 0$, we obtain $\mathscr{H}^{\tilde{s}}(Y) \leq 6^{\tilde{s}}$. Hence, $\mathscr{H}^{s}(Y)=0$ and the proof is complete.

\section{Proof of the main theorem}

Proof. Fix $x \in X$ and $n \in \mathbb{N}$. Fix $Q \in \mathscr{P}_{x}$. By $\mu_{Q}$ denote the unique invariant measure with respect to $Q$. By Lemma 2.3 choose $r_{Q, n} \in(0,1 / n)$ such that

$$
\frac{\log \mu_{Q}\left(B\left(y, r_{Q, n}\right)\right)}{\log r_{Q, n}} \leq \frac{1}{n} \quad \text { for } y \in \operatorname{supp} \mu_{Q} .
$$

Let $\tilde{r}_{Q, n} \in\left(0, r_{Q, n}\right) \cap\left(0, r_{Q, n}^{1 / n} / 3\right)$ be such that $\mu_{Q}\left(B\left(x, r_{Q, n}\right)\right) \geq 2 \tilde{r}_{Q, n}$. Choose now a number $k_{Q, n} \in \mathbb{N}$ such that

$$
2\left(1-\alpha_{Q}\right)^{k_{Q, n}} \leq \min \left\{\frac{\tilde{r}_{Q, n}^{2}}{4}, \frac{1}{3 n^{2}}\right\},
$$

where $\alpha_{Q}$ corresponds to $Q$ according to Lemma 2.3. Set

$$
d_{0}=\min \left\{\frac{\tilde{r}_{Q, n}^{2}}{4}, \frac{1}{3 n^{2}}\right\}
$$

Let $\varepsilon_{Q, n}>0$ be such that

$$
\hat{\rho}\left(P^{k_{Q, n}}, Q^{k_{Q, n}}\right)<d_{0}
$$

for every $P \in B\left(Q, \varepsilon_{Q, n}\right)$. Let $\left\{x_{m}\right\}_{m \geq 1}$ be a dense subset of $X$. Define

$$
\tilde{\mathscr{P}}=\bigcap_{m=1}^{\infty} \bigcap_{n=1}^{\infty} \bigcup_{Q \in \mathscr{P}_{x_{m}}} B\left(Q, \varepsilon_{Q, n}\right) .
$$

Since the set $\bigcup_{Q \in \mathscr{P}_{x_{m}}} B\left(Q, \varepsilon_{Q, n}\right)$ for every $m, n \in \mathbb{N}$ is open and dense, the set $\tilde{\mathscr{P}}$ is residual in $\mathscr{P}$. Fix $P \in \tilde{\mathscr{P}}$ and $m \in \mathbb{N}$. Let $\left\{Q_{n}\right\}_{n \geq 1}$ be a sequence of elements of $\mathscr{P}_{x_{m}}$ such that $P \in B\left(Q_{n}, \varepsilon_{Q, n}\right)$ for every $n \in \mathbb{N}$. For abbreviation, we set

$$
\begin{array}{ccc}
\mu_{n}=\mu_{Q_{n}}, & \alpha_{n}=\alpha_{Q_{n}}, & r_{n}=r_{Q_{n}, n}, \\
\tilde{r}_{n}=\tilde{r}_{Q_{n}, n}, & \varepsilon_{n}=\varepsilon_{Q_{n}, n}, & k_{n}=k_{Q_{n}, n} .
\end{array}
$$

Let $\mu_{1}, \mu_{2} \in M_{1}$. By Lemma 2.3 and conditions (3.2), (3.4), we have

$$
\begin{aligned}
\left\|P^{k_{n}} \mu_{1}-P^{k_{n}} \mu_{2}\right\|_{\mathscr{F}} \leq & \left\|P^{k_{n}} \mu_{1}-Q_{n}^{k_{n}} \mu_{1}\right\|_{\mathscr{F}}+\left\|Q_{n}^{k_{n}} \mu_{1}-Q_{n}^{k_{n}} \mu_{2}\right\|_{\mathscr{F}} \\
& +\left\|Q_{n}^{k_{n}} \mu_{2}-P^{k_{n}} \mu_{2}\right\|_{\mathscr{F}}<d_{0}+d_{0}+d_{0}<3 \frac{1}{3 n^{2}}=\frac{1}{n^{2}} .
\end{aligned}
$$


Since $\mu_{1}, \mu_{2} \in M_{1}$ were arbitrary and $M_{1}$ equipped with the Fortet-Mourier distance is complete, $P$ admits an invariant measure. Moreover, $P$ is asymptotically stable. Let $\mu_{*} \in$ $\mu_{1}$ be its invariant measure. First we check that $x_{m} \in \operatorname{supp} \mu_{*}$. By Lemma 2.3(iii) and the choice of $Q_{n}, n \in \mathbb{N}$, we have

$$
\begin{aligned}
\left\|\mu_{n}-\mu_{*}\right\|_{\mathscr{F}} & =\left\|Q_{n}^{k_{n}} \mu_{n}-P^{k_{n}} \mu_{*}\right\|_{\mathscr{F}} \\
& \leq\left\|Q_{n}^{k_{n}} \mu_{n}-Q_{n}^{k_{n}} \mu_{*}\right\|_{\mathscr{F}}+\left\|Q_{n}^{k_{n}} \mu_{*}-P^{k_{n}} \mu_{*}\right\|_{\mathscr{F}} \\
& \leq d_{0}+d_{0}=2 d_{0} .
\end{aligned}
$$

Fix $\varepsilon>0$. Let $n_{0} \in \mathbb{N}$ be such that $2 / n_{0}<\varepsilon$. By Lemma 2.1 and the definition of $d_{0}$, we obtain

$$
\begin{aligned}
\mu_{*}\left(B\left(x_{m}, \varepsilon\right)\right) & \geq \mu_{*}\left(B\left(x_{m}, 2 r_{n_{0}}\right)\right) \geq \mu_{*}\left(B\left(x_{m}, r_{n_{0}}+\tilde{r}_{n_{0}}\right)\right) \\
& \geq \mu_{n_{0}}\left(B\left(x_{m}, r_{n_{0}}\right)\right)-\tilde{r}_{n_{0}} \geq 2 \tilde{r}_{n_{0}}-\tilde{r}_{n_{0}}=\tilde{r}_{n_{0}} .
\end{aligned}
$$

Since $\varepsilon>0$ was arbitrary, this implies that $x_{m} \in \operatorname{supp} \mu_{*}$. By (3.8), Lemma 2.1 and the definition of $d_{0}$ we obtain

$$
\mu_{*}\left(B\left(\operatorname{supp} \mu_{n}, r_{n}^{1 / n}\right)\right) \geq \mu_{n}\left(\operatorname{supp} \mu_{n}\right)-\frac{1}{n}=1-\frac{1}{n} .
$$

Moreover, for every $y \in B\left(\operatorname{supp} \mu_{n}, r_{n}^{1 / n}\right)$, there exists $x \in \operatorname{supp} \mu_{n}$ such that

$$
B\left(x, r_{n}^{1 / n}\right) \subset B\left(y, 2 r_{n}^{1 / n}\right)
$$

and consequently by Lemma 2.1 we obtain

$$
\mu_{*}\left(B\left(y, 3 r_{n}^{1 / n}\right)\right) \geq \mu_{n}\left(B\left(y, 2 r_{n}^{1 / n}\right)\right)-\frac{r_{n}^{1 / n}}{2} \geq \mu_{n}\left(B\left(x, r_{n}^{1 / n}\right)\right)-\frac{r_{n}^{1 / n}}{2} \geq r_{n}^{1 / n}-\frac{r_{n}^{1 / n}}{2}=\frac{r_{n}^{1 / n}}{2} .
$$

Define

$$
Y=\bigcap_{m=1}^{\infty} \bigcup_{n=m}^{\infty} B\left(\operatorname{supp} \mu_{n}, r_{n}^{1 / n}\right) .
$$

By (3.10) we have $\mu_{*}(Y)=1$. On the other hand, by (3.12) we have

$$
\liminf _{r \rightarrow 0} \frac{\log \mu_{*}(B(y, r))}{\log r}=0 \quad \text { for every } y \in Y .
$$

Lemma 2.4 now shows that $\operatorname{dim}_{H}\left(\mu_{*}\right)=0$.

We have shown that $x_{m} \in \operatorname{supp} \mu_{*}$ for every $m \in \mathbb{N}$. Since $\left\{x_{m}\right\}_{m \geq 1}$ is dense, $\operatorname{supp} \mu_{*}$ is closed, and $x_{m} \in \operatorname{supp} \mu_{*}$ for every $m \in \mathbb{N}$, we obtain supp $\mu_{*}=X$. This completes the proof.

\section{Acknowledgment}

This research was supported by the State Committee for Scientific Research Grant no. Z PO3A 03125. 


\section{References}

[1] W. Bartoszek, Norm residuality of ergodic operators, Bull. Acad. Polon. Sci. Sér. Sci. Math 29 (1981), no. 3-4, 165-167.

[2] - On the residuality of mixing by convolutions probabilities, Israel J. Math. 80 (1992), no. 1-2, 183-193.

[3] J. R. Brown, Approximation theorems for Markov operators, Pacific J. Math. 16 (1966), 13-23.

[4] J. R. Choksi and S. Kakutani, Residuality of ergodic measurable transformations and of ergodic transformations which preserve an infinite measure, Indiana Univ. Math. J. 28 (1979), no. 3, 453-469.

[5] J. R. Choksi and V. S. Prasad, Approximation and Baire category theorems in ergodic theory, Measure Theory and Its Applications (Sherbrooke, Que., 1982), Lecture Notes in Math., vol. 1033, Springer, Berlin, 1983, pp. 94-113.

[6] R. M. Dudley, Probabilities and Metrics, Lecture Notes Series, vol. 45, Matematisk Institut, Aarhus Universitet, Aarhus, 1976.

[7] J. Genyuk, A typical measure typically has no local dimension, Real Anal. Exchange 23 (1997/1998), no. 2, 525-537.

[8] P. M. Gruber, Dimension and structure of typical compact sets, continua and curves, Monatsh. Math. 108 (1989), no. 2-3, 149-164.

[9] A. Iwanik, Approximation theorems for stochastic operators, Indiana Univ. Math. J. 29 (1980), no. 3, 415-425.

[10] _ Baire category of mixing for stochastic operators, Rend. Circ. Mat. Palermo (2) Suppl. (1992), no. 28, 201-217, Measure Theory Conference, (Obervolfach, 1992).

[11] A. Lasota and J. Myjak, Generic properties of stochastic semigroups, Bull. Polish Acad. Sci. Math. 40 (1992), no. 4, 283-292.

[12] _ Generic properties of fractal measures, Bull. Polish Acad. Sci. Math. 42 (1994), no. 4, 283-296.

[13] J. Myjak and R. Rudnicki, Box and packing dimensions of typical compact sets, Monatsh. Math. 131 (2000), no. 3, 223-226.

[14] J. Myjak and T. Szarek, Generic properties of Markov operators, Rend. Circ. Mat. Palermo (2) Suppl. (2002), no. 70, part II, 191-200.

[15] R. Rębowski, Most Markov operators on $C(X)$ are quasicompact and uniquely ergodic, Colloq. Math. 52 (1987), no. 2, 277-280.

[16] R. Rudnicki, Generic properties of multiplicative functions and stochastic semigroups, Bull. Polish Acad. Sci. Math. 45 (1997), no. 1, 7-16.

[17] T. Szarek, Generic properties of continuous iterated function systems, Bull. Polish Acad. Sci. Math. 47 (1999), no. 1, 77-89.

[18] _ Generic properties of learning systems, Ann. Polon. Math. 73 (2000), no. 2, 93-103.

[19] - The stability of Markov operators on Polish spaces, Studia Math. 143 (2000), no. 2, 145152.

[20] L. S. Young, Dimension, entropy and Lyapunov exponents, Ergodic Theory Dynam. Systems 2 (1982), no. 1, 109-124.

Tomasz Szarek: Institute of Mathematics, University of Silesia, Bankowa 14, 40-007 Katowice, Poland

E-mail address: szarek@itl.pl 


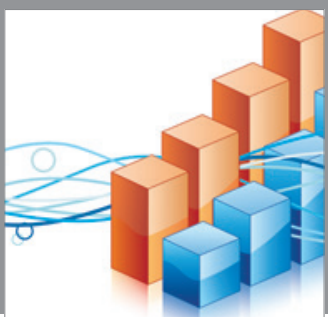

Advances in

Operations Research

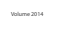

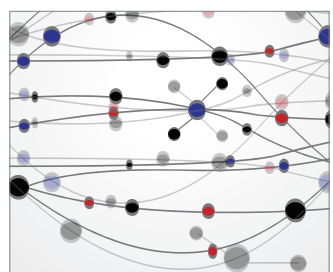

\section{The Scientific} World Journal
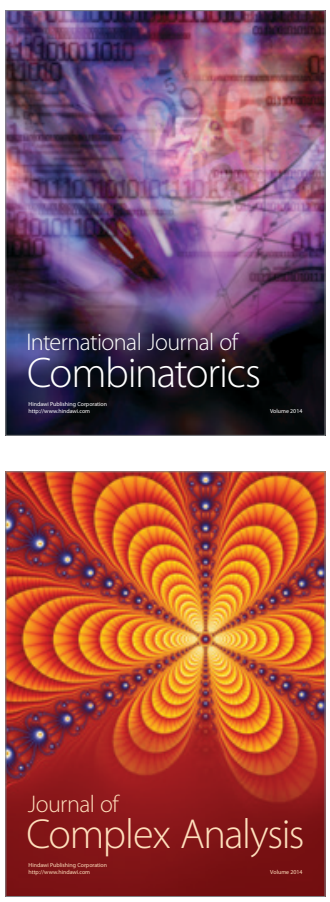

International Journal of

Mathematics and

Mathematical

Sciences
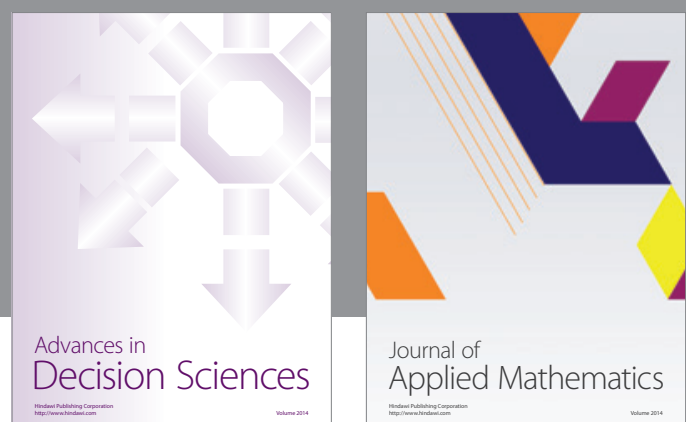

Journal of

Applied Mathematics
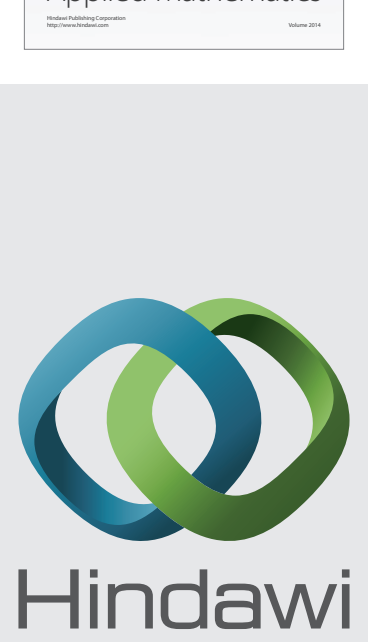

Submit your manuscripts at http://www.hindawi.com
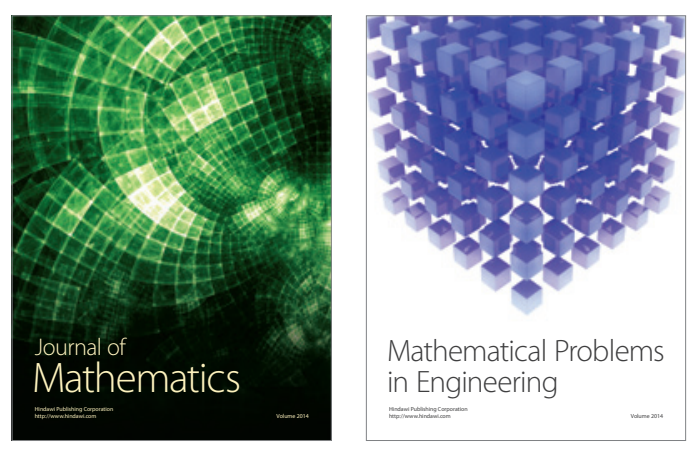

Mathematical Problems in Engineering
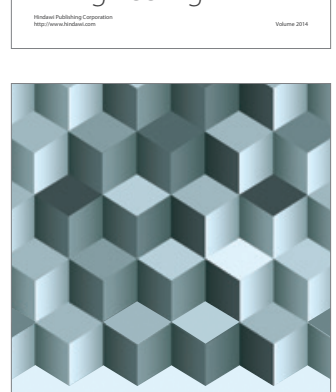

Journal of

Function Spaces
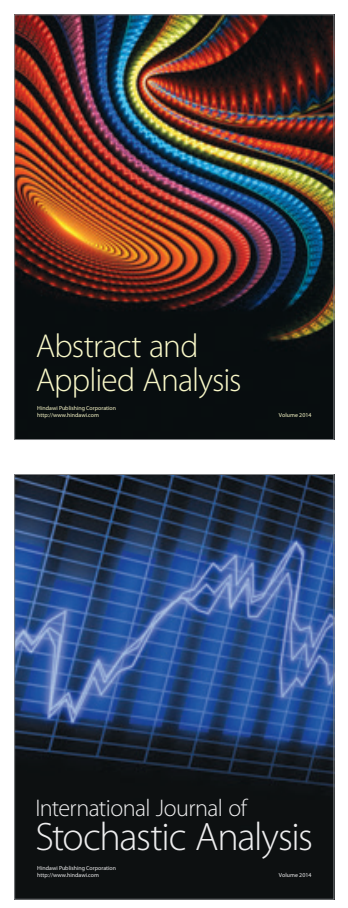

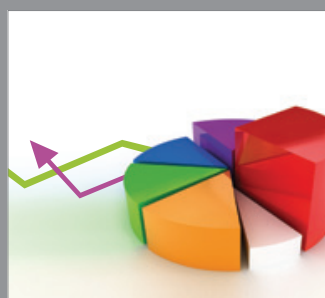

ournal of

Probability and Statistics

Promensencen
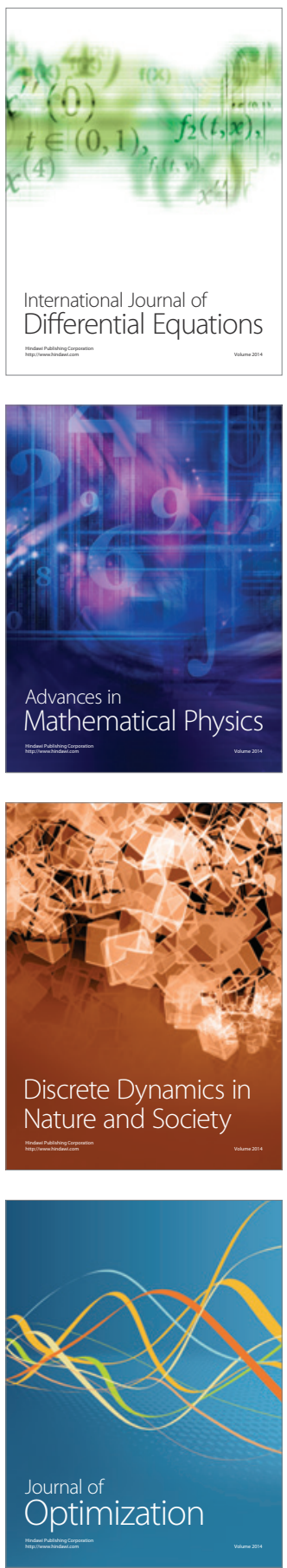